\title{
Influencia de los programas universitarios para mayores sobre la mejora del rendimiento cognitivo
}

\author{
Cristina Vilaplana Prieto \\ Departamento de Fundamentos de Análisis Económico. Universidad de Murcia, España \\ cvilaplana@um.es
}

\begin{abstract}
The aim of this paper is to apply a rigorous econometric approach to analyze the benefits derived from participation in Third Age University Programs (3AUP). Two waves from the SHARE (Survey of Health, Ageing and Retirement in Europe) corresponding to 2007 and 2011 are used. This survey contains information concerning the degree of satisfaction with life and allows to define indicators for cognitive achievement in memory, vocabulary and fluency. A sample of individuals aged between 50 and 85 years old is selected. The percentage of participants in 3AUP has increased from $3.25 \%$ to $5.47 \%$, which represents an average growth rate of $13.90 \%$. For the sample of all respondents we appreciate that: (i) the probability of retaining 10-14 words in the memory test increases by 0.34 points for participants in 3AUP, (ii) the probability of developing a list with at least twenty words increases by 0.45 points for participants in 3AUP, and it attains a maximum of 0.88 point for participants with high school education.
\end{abstract}

Keywords: Third Age University Programs, benefits, satisfaction, cognitive achievement.

\section{Resumen}

El objetivo de este trabajo es aplicar un enfoque econométrico riguroso para analizar los beneficios derivados de la participación en programas universitarios para mayores (PUM) en España. Se utilizan dos olas de la encuesta SHARE (Survey of Health, Ageing and Retirement in Europe) para 2007 y 2011. Esta encuesta contiene información sobre el nivel de satisfacción general y permite construir indicadores para pruebas cognitivas de memoria. Se selecciona una muestra de personas de entre 50 y 85 años. El porcentaje de participantes ha aumentado del 3,25\% al 5,47\%. Por tanto, en el intervalo de cuatro años la tasa de participación ha crecido a una tasa media anual acumulada del $13,90 \%$. Para el conjunto de todos los individuos se constata que: $i$ ) la probabilidad de recordar entre 10 y 14 palabras en la prueba de memoria aumenta en 0,34 puntos si participa en PUM, y ii) la probabilidad de que la lista confeccionada en la prueba de fluidez contenga 20 o más nombres aumenta en 0,45 puntos, si participa en un PUM, con un máximo de 0,88 puntos de diferencia si tiene estudios secundarios.

Palabras clave: programas universitarios para mayores, beneficios, satisfacción, rendimiento cognitivo. 


\section{Introducción}

Recientemente, se ha acuñado la expresión «envejecimiento activo» para transmitir una connotación positiva sobre la base de tres pilares: la salud, la participación y la seguridad (WHO, 2002). La idea fundamental que subyace es que independientemente de cuál sea la edad, la persona puede mantener una participación activa en cualquier ámbito, económico, social y cultural.

En España, se han llevado a cabo fructíferas investigaciones sobre las características de los programas universitarios para mayores y los beneficios derivados de estos. El análisis de Carmen et al. (2004), centrado en la Universidad de las Islas Baleares, ponía en relieve no solo la adquisición de conocimientos, sino la importancia del apoyo social recibido por los participantes. A nivel nacional, Bru (2007) realizó una evaluación de 47 programas universitarios para mayores, y señaló que los objetivos perseguidos en la mayoría de ellos son promover el conocimiento de nuevas tecnologías y el desarrollo personal. Por otra parte, Vilaplana (2010), utilizando la Encuesta de Condiciones de Vida de Personas Mayores (IMSERSO, 2006), observó que la participación en estos programas aumenta la probabilidad de considerar que la calidad de vida es buena o muy buena en un 48,99 \%. Por último, Fernández Ballesteros et al. (2012) plantearon un análisis comparativo entre participantes en programas universitarios para mayores (PUM) (grupo de tratamiento) y no participantes (grupo de control). Al final del periodo (20072010) se constató que la función cognitiva, el estado de salud y el nivel de actividad social eran significativamente superiores en el grupo de tratamiento.

El objetivo de este trabajo es contribuir a esta área de investigación aportando nuevas evidencias, con datos más actualizados y diferentes técnicas econométricas. En particular, en este trabajo se dará respuesta a la siguiente pregunta: ¿Ejerce la participación en PUM un efecto significativo sobre el rendimiento cognitivo, evaluado este a partir de pruebas de memoria y fluidez de vocabulario? La ventaja frente a otros estudios es que el tamaño de la muestra de participantes en PUM es mayor que en los anteriores trabajos mencionados, y además es posible comparar los resultados de participantes en PUM con los de no participantes.

\section{Metodología}

Se utilizan datos de la segunda y cuarta ola de la encuesta SHARE (Survey of Health, Ageing and Retirement in Europe), ${ }^{1}$ correspondientes a los años 2007 y 2011. Para el diseño de la muestra de este trabajo se ha seleccionado a individuos de entre 50 y 85 años residentes en España. Las muestras finales constan de 2003 observaciones en 2007 y 2927 observaciones en 2011.

\subsection{Definición de variables}

\section{Participación en programas universitarios para mayores}

Se define una variable binaria «PUM» que toma el valor 1 cuando ha respondido afirmativamente a esta pregunta, y cero en caso contrario. El número de participantes aumenta de 65 en 2007 a 160 en 2011, lo que en términos porcentuales supone un 3,25\% y un $5,47 \%$, respectivamente. Por tanto, la tasa de participación ha crecido a una tasa media anual acumulada del 13,90\%.

\section{Prueba de memoria}

Esta prueba consta de las siguientes fases:

- En la pantalla de un ordenador se muestra al entrevistado una lista de 10 palabras. Por ejemplo, en el cuestionario para 2007 las palabras eran: mantequilla, brazo, carta, reina, ticket, césped, esquina, piedra, libro y vara.

- Se espera un minuto y se pide al entrevistado que diga todas las palabras que recuerda.

- Se continúa con otras preguntas y, pasados unos minutos, se vuelve a pedir al entrevistado que enumere todas las palabras que recuerde.

- La puntuación total es la suma del número de palabras recordado en cada tanda, por lo que toma un valor mínimo de 0 y un valor máximo de 20 .

\section{Prueba de fluidez}

Se pide al entrevistado que enumere todos los animales que pueda en el tiempo máximo de un mi-

1 Los microdatos del SHARE son accesibles de manera gratuita a través de la página web $<$ http://www.share-project.org/> 
nuto. Se admiten como válidos todos los nombres de animales, quedando excluidos los nombres propios y las variaciones de una misma especie. La puntuación oscila entre 0 y el número máximo de animales válidos que pueda citar una persona. ${ }^{2}$

La tabla 1 muestra la información descriptiva de las personas entrevistadas en SHARE $(2007,2011)$ en función de su participación en PUM. La distribución de los no participantes está compuesta por un $47 \%$ de hombres y un $53 \%$ de mujeres, frente a un $32 \%$ de hombres y un $68 \%$ de mujeres en el grupo de participantes. Los participantes son aproximadamente cinco años más jóvenes en promedio (60 frente a 65 años). Entre los participantes hay un mayor peso de los niveles educativos secundarios y

Tabla 1. Características de la muestra en función de su participación en PUM

\begin{tabular}{|c|c|c|c|c|}
\hline & \multicolumn{2}{|c|}{2007} & \multicolumn{2}{|c|}{2011} \\
\hline & $\begin{array}{l}\text { No participa en } \\
\text { PUM }\end{array}$ & $\begin{array}{l}\text { Si participa en } \\
\text { PUM }\end{array}$ & $\begin{array}{l}\text { No participa en } \\
\text { PUM }\end{array}$ & $\begin{array}{l}\text { Sí participa en } \\
\text { PUM }\end{array}$ \\
\hline Hombre $(\%)$ & 46,80 & 32,31 & 46,98 & 33,13 \\
\hline Mujer (\%) & 53,20 & 67,69 & 53,02 & 66,88 \\
\hline Edad promedio & 65,23 & 61,28 & 64,66 & 59,52 \\
\hline \multicolumn{5}{|l|}{ Nivel de estudios (\%) } \\
\hline Sin estudios & 16,44 & 3,45 & 14,55 & 8,08 \\
\hline Educación primaria & 58,60 & 31,04 & 65,92 & 33,34 \\
\hline Educación secundaria & 11,86 & 27,59 & 8,42 & 24,24 \\
\hline Educación superior & 12,00 & 34,47 & 9,70 & 30,30 \\
\hline No indica el nivel de estudios & 1,10 & 3,45 & 1,42 & 4,04 \\
\hline \multicolumn{5}{|l|}{ Estado civil (\%) } \\
\hline Casado & 78,83 & 73,89 & 77,93 & 75,91 \\
\hline Separado & 3,81 & 4,35 & 3,46 & 4,82 \\
\hline Soltero & 7,22 & 8,70 & 4,37 & 4,82 \\
\hline Viudo & 10,14 & 13,05 & 14,25 & 14,46 \\
\hline Número promedio de enfermedades & 1,46 & 1,34 & 1,74 & 1,39 \\
\hline $\begin{array}{l}\text { Número promedio de discapacidades para activida- } \\
\text { des personales de la vida diaria }\end{array}$ & 0,22 & 0,05 & 0,37 & 0,13 \\
\hline $\begin{array}{l}\text { Número promedio de discapacidades para activida- } \\
\text { des instrumentales de la vida diaria }\end{array}$ & 0,36 & 0,11 & 0,51 & 0,14 \\
\hline \multicolumn{5}{|l|}{ Relación con la actividad económica (\%) } \\
\hline Ocupado & 20,74 & 33,85 & 14,13 & 35,63 \\
\hline Desempleado & 2,94 & 7,69 & 4,81 & 6,25 \\
\hline Incapacitado para trabajar & 4,23 & 4,62 & 3,69 & 1,88 \\
\hline Jubilado & 39,11 & 26,15 & 46,11 & 24,38 \\
\hline Labores del hogar & 31,63 & 27,69 & 29,20 & 31,25 \\
\hline Otra situación & 1,34 & 0,00 & 2,06 & 0,63 \\
\hline Ingreso anual ( $€$ constantes de 2011) & 9.639 & 10.096 & 13.950 & 14.693 \\
\hline $\begin{array}{l}\text { Prueba de memoria } \\
\text { N. }{ }^{\circ} \text { promedio de palabras recordadas } \\
\text { (mínimo: 0; máximo: 20) }\end{array}$ & 6,30 & 8,25 & 6,39 & 9,34 \\
\hline $\begin{array}{l}\text { Prueba de fluidez } \\
\text { N. . } \text { promedio de animales citados }\end{array}$ & 14,25 & 17,89 & 13,83 & 18,35 \\
\hline Número de observaciones & 1.938 & 65 & 2.767 & 160 \\
\hline
\end{tabular}

2 El número máximo de animales citados fue 39 en 2007 y 47 en 2011. 
universitarios (62,06\% en 2007 y $54,51 \%$ en 2011$)$ frente a solo $23,86 \%$ y $18,12 \%$, respectivamente, en el grupo de no participantes.

En la prueba de memoria, el número medio de palabras recordadas se encuentra en torno a 6 para no participantes, mientras que ha aumentado de 8 a 9 palabras para los participantes. En la prueba de fluidez, el número promedio de animales mencionados es superior entre los participantes, y la diferencia entre ambos grupos aumenta con el paso del tiempo (3,64 en 2007 y 4,52 animales más en promedio en 2011).

\subsection{Modelo de los resultados de las pruebas cognitivas}

Consideramos un sistema de dos ecuaciones. La primera especifica la probabilidad de que un individuo i participe en PUM en función de una serie de variables explicativas $\left(\mathrm{W}^{\prime}{ }_{\mathrm{li}}\right)$. La segunda ecuación especifica la variable cognitiva (fluidez o vocabulario) en función de una serie de variables explicativas $\left(\mathrm{W}_{2 \mathrm{i}}\right.$ ) y de la participación en PUM.

$$
\begin{aligned}
& \operatorname{PUM}_{\mathrm{i}}^{*}=\mathrm{W}_{1 \mathrm{i}}{ }_{1 \mathrm{\alpha}} \alpha_{1}+\theta_{1 \mathrm{i}}(1) \\
& \text { Cognitiva }_{\mathrm{i}}{ }^{*}=\gamma \mathrm{PUM}_{\mathrm{i}}^{*}+\mathrm{W}_{2 \mathrm{i}}{ }_{2 \mathrm{i}} \alpha_{2}+\theta_{2 \mathrm{i}}(2) \\
& \text { Cognitiva }_{\mathrm{i}}{ }^{*}=\left\{\text { Memoria }_{\mathrm{i}}{ }, \text { Fluidez }_{\mathrm{i}}^{*}\right\}
\end{aligned}
$$

Los resultados de las pruebas cognitivas siempre toman valores no negativos y muestran una distribución con un perfil decreciente. Esta característica determina que el modelo que debemos estimar para los resultados de las pruebas cognitivas es de tipo Poisson. En ambos vectores de variables explicativas $\left(\mathrm{W}^{\prime}{ }_{1 \mathrm{i}} \mathrm{y} \mathrm{W}^{\prime}{ }_{2 \mathrm{i}}\right.$ ) se incluyen edad, género, nivel de estudios, estado de salud (enfermedades crónicas y discapacidades), y relación con la actividad económica. Actuando como restricciones de identificación, y por tanto solamente en $\mathrm{W}^{\prime}{ }_{2 \mathrm{i}}$ se incluyen estado civil, cuidar a otras personas, tamaño del municipio de residencia, y nivel de ingresos. La filosofía que hay detrás de estas variables es que la disponibilidad de tiempo, las circunstancias económicas y geográficas pueden afectar a la motivación de iniciar un PUM.

\section{Resultados y discusión}

A partir de las estimaciones de los modelos para las pruebas cognitivas se han obtenido las probabilidades predichas. La tabla 2 muestra los resultados de las probabilidades predichas para la prueba de memoria. Para el conjunto de la muestra, la probabilidad de que el entrevistado recuerde entre 10 y 14 palabras aumenta en 0,34 puntos si participa en PUM. La probabilidad de recordar entre 10 y 14 palabras para los participantes en PUM es superior a la de los no participantes cuando comparamos personas de 50 a 59 años (0,55 puntos), estudios secundarios $(0,91$ puntos $)$ y estudios superiores $(0,64$ puntos). En el grupo de personas de 80 y más años, aunque no hay ningún caso que haya recordado más de 10 palabras, hay que valorar muy positivamente el aumento en la probabilidad de recordar entre $5 \mathrm{y}$ 9 palabras cuando se ha participado en PUM $(0,78$ puntos en el grupo de 80 y más años; 0,55 puntos en el grupo de sin estudios).

Los resultados de las probabilidades predichas para la prueba de fluidez se ofrecen en la tabla 3 . La probabilidad de que la lista de animales que debían confeccionar los entrevistados contenga 20 o más nombres aumenta en 0,45 puntos si participa en un PUM, con un máximo de 0,88 puntos de diferencia si tiene estudios secundarios y 0,80 puntos si tiene estudios superiores. Al igual que en la prueba de memoria, se aprecia que la probabilidad de recordar un mayor número de nombres de animales disminuye a medida que aumenta la edad, pero incluso en este caso, la probabilidad de recordar entre 14 y 19 nombres aumenta sustancialmente para los participantes en PUM (0,96 puntos para el grupo de 70-79 años; 0,80 puntos para el grupo de 80 y más años). 
DOI: HTTP://DX.DOI.ORG/10.6035/ARIADNA.2016.3.10 - ISSN: 2340-7719 VOL. I - ISSUE 3, pP 71-77

Tabla 2. Probabilidad predicha para la prueba de memoria. Probabilidad de recordar un número determinado de palabras de la lista propuesta por el entrevistador condicionado a participar o no en PUM

\begin{tabular}{|c|c|c|c|}
\hline & $\begin{array}{l}\text { No participa en } \\
\text { PUM (1) }\end{array}$ & $\begin{array}{l}\text { Si participa } \\
\text { en } P U M(2)\end{array}$ & $\begin{array}{c}\text { Diferencia entre las probabilidades } \\
\text { predichas (2) - (1) }\end{array}$ \\
\hline \multicolumn{4}{|l|}{ Toda la muestra } \\
\hline De 0 a 4 palabras & 0,18 & 0,00 & $-0,18$ \\
\hline De 5 a 9 palabras & 0,81 & 0,65 & $-0,16$ \\
\hline De 10 a 14 palabras & 0,00 & 0,34 & 0,34 \\
\hline \multicolumn{4}{|l|}{ Edad: De 50 a 59 años } \\
\hline De 0 a 4 palabras & 0,01 & 0,00 & $-0,01$ \\
\hline De 5 a 9 palabras & 0,98 & 0,44 & $-0,55$ \\
\hline De 10 a 14 palabras & 0,01 & 0,56 & 0,55 \\
\hline \multicolumn{4}{|l|}{ Edad: De 60 a 69 años } \\
\hline De 0 a 4 palabras & 0,03 & 0,00 & $-0,03$ \\
\hline De 5 a 9 palabras & 0,97 & 0,95 & $-0,01$ \\
\hline De 10 a 14 palabras & 0,00 & 0,05 & 0,05 \\
\hline \multicolumn{4}{|l|}{ Edad: De 70 a 79 años } \\
\hline De 0 a 4 palabras & 0,37 & 0,00 & $-0,37$ \\
\hline De 5 a 9 palabras & 0,63 & 0,93 & 0,30 \\
\hline De 10 a 14 palabras & 0,00 & 0,07 & 0,07 \\
\hline \multicolumn{4}{|l|}{ Edad: 80 o más años } \\
\hline De 0 a 4 palabras & 0,97 & 0,20 & $-0,77$ \\
\hline De 5 a 9 palabras & 0,02 & 0,80 & 0,78 \\
\hline De 10 a 14 palabras & 0,00 & 0,00 & 0,00 \\
\hline \multicolumn{4}{|l|}{ Estudios: Primarios } \\
\hline De 0 a 4 palabras & 0,19 & 0,00 & $-0,19$ \\
\hline De 5 a 9 palabras & 0,81 & 0,86 & 0,05 \\
\hline De 10 a 14 palabras & 0,00 & 0,14 & 0,14 \\
\hline \multicolumn{4}{|l|}{ Estudios: Secundarios } \\
\hline De 0 a 4 palabras & 0,00 & 0,00 & 0,00 \\
\hline De 5 a 9 palabras & 1,00 & 0,09 & $-0,91$ \\
\hline De 10 a 14 palabras & 0,00 & 0,91 & 0,91 \\
\hline \multicolumn{4}{|l|}{ Estudios: Superiores } \\
\hline De 0 a 4 palabras & 0,06 & 0,00 & $-0,06$ \\
\hline De 5 a 9 palabras & 0,88 & 0,30 & $-0,58$ \\
\hline De 10 a 14 palabras & 0,06 & 0,70 & 0,64 \\
\hline
\end{tabular}


Tabla 3. Probabilidad predicha para la prueba de fluidez. Probabilidad de citar un número determinado de animales condicionado a participar o no en PUM

\begin{tabular}{|c|c|c|c|}
\hline & $\begin{array}{l}\text { No participa en } \\
\text { PUM (1) }\end{array}$ & $\begin{array}{l}\text { Si participa } \\
\text { en PUM (2) }\end{array}$ & $\begin{array}{c}\text { Diferencia entre las probabilidades } \\
\text { predichas (2) - (1) }\end{array}$ \\
\hline \multicolumn{4}{|l|}{ Toda la muestra } \\
\hline De 0 a 4 palabras & 0,00 & 0,00 & 0,00 \\
\hline De 5 a 9 palabras & 0,06 & 0,00 & $-0,05$ \\
\hline De 10 a 14 palabras & 0,61 & 0,00 & $-0,61$ \\
\hline De 15 a 19 palabras & 0,33 & 0,55 & 0,21 \\
\hline 20 o más palabras & 0,00 & 0,45 & 0,45 \\
\hline \multicolumn{4}{|l|}{ Edad: De 50 a 59 años } \\
\hline De 0 a 4 palabras & 0,00 & 0,00 & 0,00 \\
\hline De 5 a 9 palabras & 0,01 & 0,00 & $-0,01$ \\
\hline De 10 a 14 palabras & 0,31 & 0,00 & $-0,31$ \\
\hline De 15 a 19 palabras & 0,68 & 0,28 & $-0,40$ \\
\hline 20 o más palabras & 0,00 & 0,72 & 0,72 \\
\hline \multicolumn{4}{|l|}{ Edad: De 60 a 69 años } \\
\hline De 0 a 4 palabras & 0,00 & 0,00 & 0,00 \\
\hline De 5 a 9 palabras & 0,02 & 0,00 & $-0,02$ \\
\hline De 10 a 14 palabras & 0,66 & 0,00 & $-0,66$ \\
\hline De 15 a 19 palabras & 0,32 & 0,87 & 0,55 \\
\hline 20 o más palabras & 0,00 & 0,13 & 0,13 \\
\hline \multicolumn{4}{|l|}{ Edad: De 70 a 79 años } \\
\hline De 0 a 4 palabras & 0,00 & 0,00 & 0,00 \\
\hline De 5 a 9 palabras & 0,09 & 0,00 & $-0,09$ \\
\hline De 10 a 14 palabras & 0,90 & 0,00 & $-0,90$ \\
\hline De 15 a 19 palabras & 0,01 & 0,97 & 0,96 \\
\hline 20 o más palabras & 0,00 & 0,03 & 0,03 \\
\hline \multicolumn{4}{|l|}{ Edad: 80 o más años } \\
\hline De 0 a 4 palabras & 0,04 & 0,00 & $-0,04$ \\
\hline De 5 a 9 palabras & 0,34 & 0,20 & $-0,14$ \\
\hline De 10 a 14 palabras & 0,61 & 0,00 & $-0,61$ \\
\hline De 15 a 19 palabras & 0,00 & 0,80 & 0,80 \\
\hline 20 o más palabras & 0,00 & 0,00 & 0,00 \\
\hline \multicolumn{4}{|l|}{ Estudios: Primarios } \\
\hline De 0 a 4 palabras & 0,00 & 0,00 & 0,00 \\
\hline De 5 a 9 palabras & 0,06 & 0,00 & $-0,06$ \\
\hline De 10 a 14 palabras & 0,67 & 0,00 & $-0,67$ \\
\hline De 15 a 19 palabras & 0,27 & 0,71 & 0,45 \\
\hline 20 o más palabras & 0,00 & 0,29 & 0,29 \\
\hline \multicolumn{4}{|l|}{ Estudios: Secundarios } \\
\hline De 0 a 4 palabras & 0,00 & 0,00 & 0,00 \\
\hline De 5 a 9 palabras & 0,00 & 0,00 & 0,00 \\
\hline De 10 a 14 palabras & 0,14 & 0,00 & $-0,14$ \\
\hline De 15 a 19 palabras & 0,86 & 0,13 & $-0,74$ \\
\hline 20 o más palabras & 0,00 & 0,88 & 0,88 \\
\hline
\end{tabular}




\begin{tabular}{|c|c|c|c|}
\hline & $\begin{array}{c}\text { No participa en } \\
\text { PUM (1) }\end{array}$ & $\begin{array}{c}\text { Si participa } \\
\text { en PUM (2) }\end{array}$ & $\begin{array}{c}\text { Diferencia entre las probabilidades } \\
\text { predichas (2) }-(1)\end{array}$ \\
\hline Estudios: Superiores & & & 0,00 \\
\hline De 0 a 4 palabras & 0,00 & 0,00 & $-0,01$ \\
\hline De 5 a 9 palabras & 0,01 & 0,00 & $-0,25$ \\
\hline De 10 a 14 palabras & 0,25 & 0,00 & $-0,54$ \\
\hline De 15 a 19 palabras & 0,74 & 0,20 & 0,80 \\
\hline 20 o más palabras & 0,00 & 0,80 & \\
\hline
\end{tabular}

\section{Conclusiones}

En esta sección se propone ofrecer una serie de recomendaciones sobre cómo orientar los PUM de cara al futuro. En primer lugar, conviene recordar el trabajo de Duay y Brian (2008) que, a partir de entrevistas a participantes en PUM, concluyeron que el diseño de programas de aprendizaje efectivos debía sustentarse sobre las siguientes bases: 1) que los temas que se fuesen a tratar resultasen interesantes; 2) que el profesor se sintiese implicado con el tema de los programas universitarios para mayores, y 3) que las experiencias de aprendizaje permitiesen la implicación de los estudiantes, es decir, que se fomentase una actitud muy participativa. En segundo lugar, es primordial construir un aprendizaje bidireccional, en el que las personas mayores compartan su saber y sus habilidades con las generaciones más jóvenes, y estas a su vez les transmitan sus propios conocimientos e innovaciones. Si esto llegara a suceder, conseguiríamos una sociedad más inclusiva, donde todas las formas de aprendizaje serían valoradas, se apreciaría la contribución de las personas mayores como un elemento más del sistema educativo y se reconocerían los beneficios del aprendizaje a lo largo de toda la vida.

\section{Referencias}

Bru, C. (2007). Older Adults University Programs in Spain. Proceedings of the International Conference on Learning in Later Life. Glasgow: Senior Studies Institute. University of Strathclyde (pp. 29-38).

Carmen, O. S., Luis, B. B. \& Carmen, T. G. (2004). University programs for seniors in Spain. Educational Gerontology, 30, 315-328.

Duay, D. \& Bryan, V. (2008). Learning in later life what seniors want in a learning experience. Educational Gerontology; 34 (12).

Fernández-Ballesteros, R., Molina, M.A., Schettini, R., \& Rey, A.L., (2012). Promoting active aging through University Programs for Older Adults. The Journal of Gerontopsychology and Geriatric Psychiatry, 25, 145-154.

IMSERSO (2006). A propósito de las condiciones de vida de las personas mayores.

Vilaplana Prieto, C. (2010). Relación entre los Programas Universitarios para Mayores, la satisfacción durante la jubilación y la calidad de vida. Revista de Investigación Educativa, 28, 195-216.

World Health Organization (2002). Active aging: a policy framework. Geneva: World Health Organization. 